\title{
Primera cita en la península Ibérica del poliqueto terrestre Parergodrilus heideri Reisinger, 1925 (Polychaeta, Parergodrilidae)
}

\author{
E. Martínez-Ansemil ${ }^{1} \&$ J. Parapar ${ }^{1}$
}

Los Parergodrílidos (Polychaeta, Parergodrilidae) son un pequeño grupo de anélidos de cuerpo diminuto y cilíndrico, de organización corporal sencilla, sin apéndices y probable origen progenético (Giere, 2009), inicialmente considerados como 'arquianélidos' hermafroditas o como oligoquetos enquitreidos, pero posteriormente reconocidos como poliquetos en diversos trabajos tanto de índole morfológica como molecular (e.g. Karling, 1958; Reisinger, 1960; Rota et al., 2001; Struck et al., 2002; McHugh, 2005; Rousset et al., 2007).

La familia se compone de dos especies, Parergodrilus heideri Reisinger, 1925, de hábitos terrestres y Stygocapitella subterranea Knöller, 1934, habitante de arenas de niveles altos de playa y durante mucho tiempo considerada un oligoqueto enquitreido.

Parergodrilus heideri, junto con Hrabeiella periglandulata Pizl \& Chalupský, 1984, constituyen los dos únicos ejemplos conocidos de poliquetos de hábitos terrestres, a los que se añaden algunas especies de nereidos encontradas, en contadas ocasiones, en suelos tropicales (Glasby y Timm, 2008). Sus peculiaridades anatómicas y modo de vida terrestre o de transición marinoterrestre hacen de $P$. heideri, $S$. subterranea y $H$. periglandulata tres especies de gran relevancia en el estudio sistemático y filogenético de anélidos.

La posición filogenética de los parergodrílidos es todavía incierta, pudiendo considerarse como autapomorfías del taxón la singular organización estructural de la faringe y la presencia de glándulas especiales que utilizan presumiblemente para la formación de las cápsulas que rodean a los huevos (Purschke, 1987, 1999). Sin embargo Westheide (1990) destaca diferencias en la faringe de ambos géneros que pueden hacer dudar sobre su carácter homólogo. A diferencia de P. heideri, S. subterranea probablemente represente un complejo de especies morfológicamente similares (Schmidt \& Westheide, 2000; Giere, 2009).

Con ocasión del proyecto PASCALIS (Protocols for the ASsesment and Conservation of Aquatic Life In the Subsurface), se ha obtenido un ejemplar de $P$. heideri a partir de una muestra de agua del medio hiporreico de la ribera del río Bustablado (Arredondo, Cantabria). Para la toma de muestras se siguió el método de Karaman-Chappuis, consistente en la excavación de un hoyo en el borde del río y el filtrado del agua intersticial que en él se concentra. La muestra fue fijada en formaldehído al $4 \%$ y el material biológico conservado en etanol al 70\% hasta su identificación.

Presentamos a continuación un breve resumen de las principales características anatómicas que permiten el reconocimiento de la especie. Las observaciones, mediciones y fotografías han sido realizadas con un microscopio NIKON 90I dotado de contraste interferencial.

El ejemplar se encuentra depositado en el Museo Nacional de Ciencias Naturales de Madrid (MNCN).

\section{Parergodrilus heideri Reisinger, 1925}

Parergodrilus heideri Reisinger, 1925: 197-254, figs 1-15, pl. 1. Rota, 1997: 92-94, figs 2-6. Rota, 1998: 77-82, figs 1-16.

MATERIAL EXAMINADO. MNCN 16.01/3007. Un individuo, recolectado el 28 de enero de 2003, montaje in toto en Bálsamo del Canadá. Localidad: ribera del río Bustablado (Arredondo, Cantabria); coordenadas en grados decimales: $-3,104388889$ y 43,28122222; altitud $147 \mathrm{~m}$.

Departamento de Bioloxía Animal, Bioloxía Vexetal e Ecoloxía, Facultade de Ciencias, Universidade da Coruña, Alejandro de la Sota 1, 15008 A Coruña eansemil@udc.es y jparapar@udc.es 

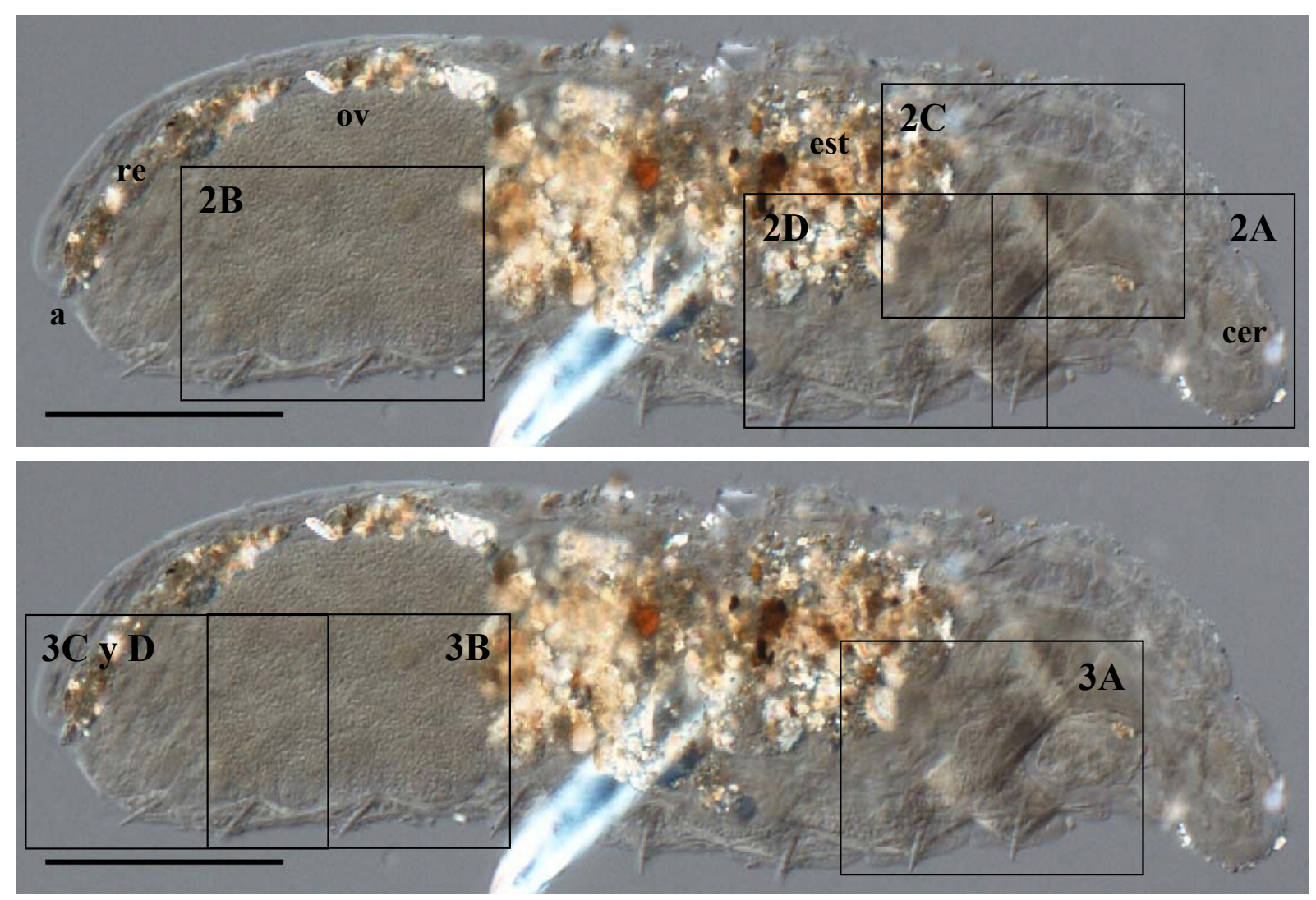

Fig. 1.- Parergodrilus heideri. Vista lateral derecha del ejemplar encontrado en Cantabria (MNCN 16.01/3007). Se destacan los elementos corporales más conspicuos y se enmarcan las zonas corporales mostradas en detalle en las figuras 2 y 3 . a = ano; cer $=$ cerebro; est $=$ estómago; ov = ovocito; re $=$ recto. Escalas $100 \mu \mathrm{m}$.

Fig. 1.- Parergodrilus heideri. Lateral right view of the specimen found in Cantabria (MNCN 16.01/3007). The most conspicuous components of the body are detached and framed body regions are shown in figures 2 and 3. $\mathrm{a}=$ anus; cer $=$ brain; est $=$ stomach; ov = egg; re = rectum. Scales $100 \mu \mathrm{m}$.

A diferencia de la gran mayoría de los poliquetos, en los cuales la taxonomía se basa fundamentalmente en caracteres morfológicos, en los parergodrílidos y otras familias o géneros de poliquetos de pequeño tamaño con escasos caracteres externos de relevancia (Trilobodrilus, Dinophilus, Diurodrilus, etc.), su estudio taxonómico se sustenta fundamentalmente en caracteres anatómicos internos de más o menos fácil observación gracias a su cuerpo transparente.

Parergodrilus heideri está muy bien caracterizada desde su descripción original por Reisinger (1925) quien realiza un profundo estudio de su anatomía interna. Recientemente las publicaciones de Rota $(1997,1998)$ han venido a complementar la descripción original al realizar una redescripción del taxón que reúne además las observaciones de Reisinger y de otros autores. Por ello, a continuación nos limitaremos a destacar únicamente aquellos aspectos más fácilmente observables de la anatomía interna del ejemplar encontrado en Cantabria, que permiten identificarlo sin ningún género de dudas como $P$. heideri.

DESCRIPCIÓN DEL EJEMPLAR. Cuerpo corto, cilíndrico y no regionalizado, de $520 \mu \mathrm{m}$ de longitud y alrededor de $125 \mu \mathrm{m}$ de anchura (Fig. 1).

Se trata de una hembra adulta que, como es característico en la especie, está dotada de prostomio, peristomio, ocho segmentos setígeros y pigidio reducido.

Superficie corporal lisa, con una capa cuticular transparente (Fig. 3D, c), de hasta $5 \mu \mathrm{m}$ de grosor 

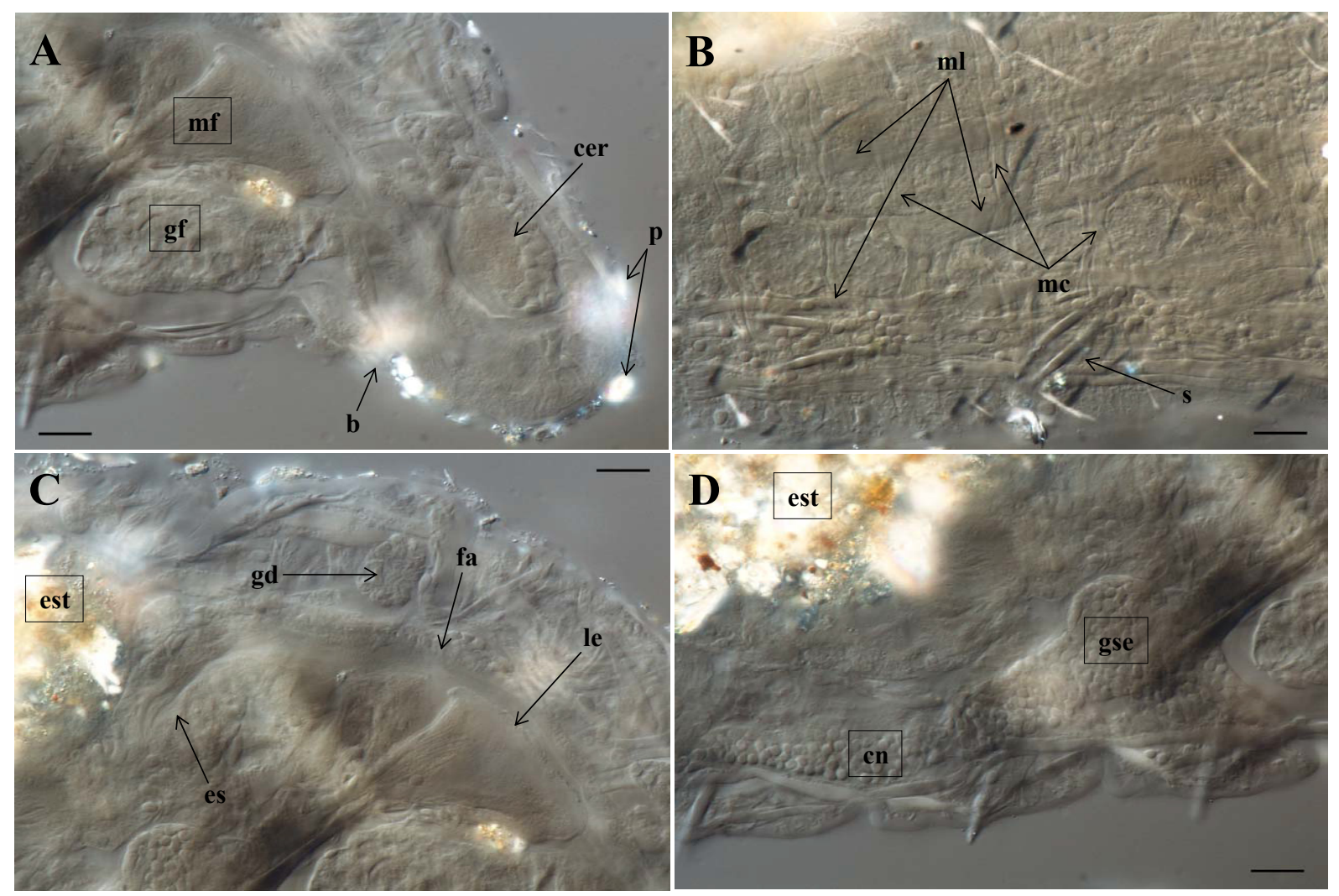

Fig. 2.- Parergodrilus heideri. A. Región anterior (prostomio, peristomio y primer segmento setígero). B. Entramado de paquetes musculares en la pared corporal. C. Región antero-dorsal. D. Región antero-ventral. $\mathrm{b}=$ boca; cer $=$ cerebro; $\mathrm{cn}=\mathrm{cadena}$ nerviosa; es = esófago; est = estómago; fa = faringe; $\mathrm{gd}=$ glándula dorsal; $\mathrm{gf}$ = glándula faríngea; gse = ganglio subesofágico; $\mathrm{mc}=$ paquetes musculares circulares; $\mathrm{mf}=$ músculo faríngeo, $\mathrm{ml}=$ paquetes musculares longitudinales; $\mathrm{p}=$ partículas adheridas al cuerpo; $\mathrm{s}=$ sedas. Escalas $10 \mu \mathrm{m}$.

Fig. 2.- Parergodrilus heideri. Anterior region (prostomium, peristomium and first chaetigerous segment). B. Network of muscular bundles in body wall. C. Antero-dorsal region. D. Antero-ventral region. B = mouth; cer = brain; $\mathrm{cn}=$ nerve cord; es = oesophagus; $\mathrm{est}=$ stomach; $\mathrm{fa}=$ pharynx; $\mathrm{gd}=$ dorsal gland; $\mathrm{gf}=$ pharyngeal gland; gse $=$ sub-oesophageal ganglion; $\mathrm{mc}=$ circular muscle fibres; $\mathrm{mf}=$ pharyngeal muscle; $\mathrm{ml}=$ longitudinal muscle fibres; $\mathrm{p}=$ particles adhered to the body; $\mathrm{s}=$ setae. Scales $10 \mu \mathrm{m}$.

(extremo posterior del cuerpo), a la que se hallan adheridas algunas partículas finas del sedimento (Fig. 2A, p). A través de la cutícula y de una fina epidermis es posible observar en la pared corporal la presencia de paquetes musculares longitudinales y circulares que se entrecruzan y dan al animal un peculiar aspecto reticulado (Fig. $2 \mathrm{~B}, \mathrm{ml}, \mathrm{mc}$ ). Dos pares de sedas ventrales por segmento setígero (27$31 \mu \mathrm{m}$ de longitud), con forma de barra engrosada, curvada en su base y ligeramente afilada hacia su extremo distal (Figs. 2B y D, s).

En la pared corporal desembocan varios pares de glándulas epidérmicas dorsales con disposición segmentaria (Fig. 2C, gd) y un único par de glán- dulas epidérmicas ventrales en las proximidades de los haces de sedas del primer segmento setígero (Fig. 3A, gv).

La cavidad bucal conduce a una faringe no ciliada (Fig. 2C, fa), provista de un área basal revestida por una gruesa cutícula ('lengua') (Fig. 2C, le), que puede ser proyectada al exterior y luego retraída gracias a la acción de un complejo sistema muscular (Fig. 2A, mf). Asociada al proceso de alimentación, destaca también la presencia de una voluminosa glándula que desemboca en la cara ventral del conducto bucal, antes de la lengua (Fig. 2A, gf). Un corto esófago ciliado (Fig. 2C, es) da paso a un amplio estómago cilíndrico (Figs. 1 y $2 \mathrm{C}$, 

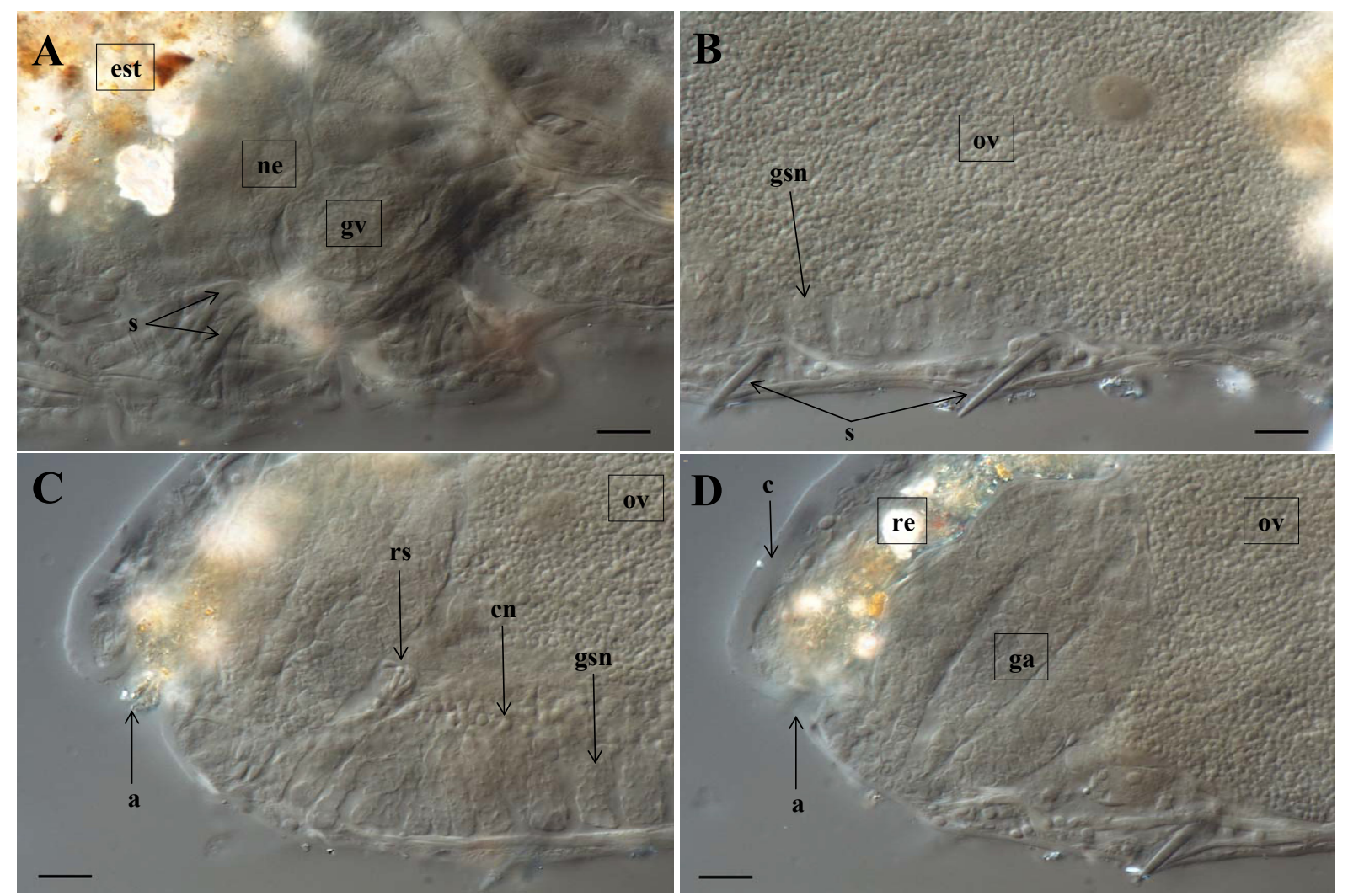

Fig. 3.- Parergodrilus heideri. A. Región antero-ventral. B. Región medio-posterior. C y D. Región posterior. a $=$ ano; $\mathrm{c}=$ cutícula; $\mathrm{cn}=$ cadena nerviosa; est = estómago; ga = glándula adanal; gsn = glándula subneural; gv = glándula ventral; ne = metanefridio; ov = ovocito; re = recto; $r \mathrm{~s}=$ receptáculo seminal; $\mathrm{s}=$ sedas. Escalas $10 \mu \mathrm{m}$.

Fig. 3.- Parergodrilus heideri. A. Antero-ventral region. B. Middle-posterior region. C and D. Posterior region. $\mathrm{a}=$ anus; $\mathrm{c}=$ cuticle; $\mathrm{cn}=$ nerve cord; est $=$ stomach; ga = adanal gland; gsn = subneural gland; gv = ventral gland; ne = metanephridium; ov = egg; re = rectum; $\mathrm{rs}=$ seminal receptacle; $\mathrm{s}=$ setae. Scales $10 \mu \mathrm{m}$.

est) el cual se continúa en un largo y sinuoso intestino ciliado, que se continúa en un recto no ciliado que discurre justo bajo la pared corporal dorsal (Fig. 3D, re) y desemboca en un ano subterminal (Figs. 1, 3C y D, a).

Dos pares de metanefridios se abren en la cara ventral del segundo y tercer segmentos setígeros (Fig. 3A, ne).

El cerebro se localiza confinado en el prostomio. (Fig. 2A, cer), y conecta con la cadena nerviosa ventral por medio de un par de conectivos circunfaríngeos que se reúnen en un ganglio subesofágico (Fig. 2D, gse). La cadena nerviosa ventral discurre próxima a la epidermis (Fig. 2D, cn) hasta la región caudal, donde se separa de la pared corporal para localizarse por encima de una voluminosa masa glandular asociada al área genital (glándulas subneurales) (Figs. 3B y C, cn, gsn).

Los ovarios se localizan al fondo de un saco ovárico en el sexto y séptimo segmentos setígeros (Fig. 1). Los voluminosos ovocitos maduros localizados en su interior (Figs. 3B-D, ov) son conducidos al exterior por oviductos que abren en un par de poros ventrales. Asociado al tramo final de cada oviducto se observa un receptáculo seminal a modo de divertículo esférico (Fig. 3C, rs). Dos grandes glándulas denominadas 'glándulas adanales' (Fig. $3 \mathrm{D}$, ga) ocupan la mayor parte del último segmento setígero y se abren al exterior en las proximidades de los poros genitales femeninos.

HÁBITAT. Especie hallada en suelos húmedos, hojarasca y tocones en descomposición principal- 
mente en bosques de hayas $\mathrm{y}$, menos frecuentemente, en y junto a riachuelos y charcas de agua dulce (Graefe, 1993; Glasby, 2000). El ejemplar hallado en Cantabria proviene del medio hiporreico próximo al cauce del río Bustablado. En la misma muestra se han identificado los enquitreidos anfibios Henlea perpusilla Friend, 1911, Marionina argentea (Michaelsen, 1889) y Cernosvitoviella spp. (comúnmente encontradas en aguas subterráneas) y se ha detectado la presencia de un naídido tubificíneo dotado de sedas capilares (fauna típicamente acuática) que no ha podido ser identificado por no hallarse en estado de madurez sexual.

DistribuCIÓN GEOGRÁfiCA. Tras su descripción original, $P$. heideri ha sido localizado en otros países europeos como Alemania (Remane, 1932; Graefe, 1977; 1993; Schwank, 1981; Purschke, 1987; Römbke \& Jans, 1991), Francia (Remy, 1935; Schweitzer, 1936), Croacia (Reisinger, 1960), Suecia y República Checa (Chalupský, 1992) y más recientemente en Italia por Rota (1997).

La presente cita en Cantabria representa el primer registro del taxon al otro lado de los Pirineos así como su hallazgo más meridional.

Discusión. Las características del ejemplar recogido en Cantabria se corresponden muy bien con las descripciones que existen de esta especie. El hecho de que el ejemplar encontrado se trate de una hembra no es extraño pues varios autores han destacado la dificultad de encontrar machos (e.g. Reisinger, 1960; Chalupský, 1992). En este sentido es de destacar la observación de Rota (1997) de hembras conteniendo esperma en sus receptáculos seminales, a pesar de la ausencia de machos en sus muestras.

La existencia en machos de 9 segmentos setígeros por la presencia de un segmento setígero adicional portador de sedas genitales asociadas a un atrio genital impar subterminal, hace que exista un cierto dimorfismo sexual en la especie.

Dada la amplia presencia de $P$. heideri por toda Europa Central, no es de extrañar la presencia de esta especie en la zona norte de la península Ibérica.

Es muy posible que esta especie se encuentre mucho más repartida por ambientes semejantes de la cornisa cantábrica, habiendo pasado hasta ahora desapercibida su presencia debido a su diminuto tamaño y posible confusión con un oligoqueto enquitreido.

\section{AGRADECIMIENTOS}

Este estudio fue financiado con cargo al proyecto europeo PASCALIS (EVK2CT-2001-00121). Los autores agradecen a la Dra. Ana Camacho (responsable del proyecto por España) y a su grupo de investigación del Museo Nacional de Ciencias Naturales de Madrid por la toma de muestras y la separación del material.

\section{Referencias}

ChalupskÝ, J., 1992. Terrestrial Enchytraeidae (Oligochaeta) and Parergodrilidae from Sweden, with description of a new enchytraeid species. Zoologica Scripta, 21: 133-150.

GIERE, O., 2009. Meiobenthology. The microscopic motile fauna of aquatic sediments. 2nd edition. SpringerVerlag. Berlin. $527 \mathrm{pp}$.

Glasby, C., 2000. Family Parergodrilidae. In: P. L. Beesley, G. J. B. Ross, \& C. J. Glasby (eds). Polychaetes \& Allies: The Southern Synthesis. Fauna of Australia, vol. 4a Polychaeta, Myzostomida, Pogonophora, Echiura, Sipuncula. CSIRO. Melbourne: 240-241.

GlasBy , C. \& Timm, T., 2008. Global diversity of polychaetes (Polychaeta; Annelida) in freshwater. Hydrobiologia, 595: 107-115.

Graefe, U., 1977. Adenodrilus Graefe (in press) and Parergodrilus Reisinger, two aberrant Annelida in forest soils. Publicaciones del Centro pirenaico de Biología Experimental, 9: 25.

GraEfe, U., 1993. Die Gliederung von Zersetzergesellschaften für die standortsökologische Ansprache. Mitteilungen der Deutschen Bodenkundlichen Gesellschaft, 69: 95-98.

KARLING, T. G., 1958. Zur Kenntnis von Stygocapitella subterranea Knöller und Parergodrilus heideri Reisinger (Annelida). Arkiv fur Zoology, 11: 307-341.

McHugh, D., 2005. Molecular systematics of polychaetes (Annelida). Hydrobiologia, 535/536: 309-318.

PuRsChKe, G., 1987. Anatomy and ultrastructure of ventral pharyngeal organs and their phylogenetic importance in Polychaeta (Annelida). III. The pharynx of the Parergodrilidae. Zoologische Jahrbücher, Anatomie, 115: 331-362.

PurschKe, G., 1999. Terrestrial polychaetes - models for the evolution of the Clitellata (Annelida)? Hydrobiologia, 406: 87-99.

REISINGER, E., 1925. Ein landbewohnender Archiannelide. (Zugleich ein Beitrag zur Systematik der Archianneliden). Zeitschrift für Morphologie und Ökologie der Tiere, 3: 197-254.

ReISINGER, E., 1960. Die Lösung des ParergodrilusProblems. Zeitschrift für Morphologie und Ökologie der Tiere, 48: 517-544. 
Remane, A., 1932. Archiannelida. In: G. Grimpe \& E. Wagler (eds). Tierwelt der Nord und Ostsee, 6: 1-36.

Remy, P., 1935. Quelques Pauropodes de France et des Balkans. Bulletin de la Société d'Histoire Naturelle de la Moselle, 10: 273-294.

RömBKE, J. \& JANS, W., 1991. On terrestrial Polychaeta (Annelida) from forest soils of southern Germany. Verhandlungen der Deutschen Zoologischen Gesellschaft, 84: 447-448.

RoTA, E., 1997. First italian record of the terrestrial polychaete Parergodrilus heideri Reisinger, with anatomical an ecological notes. Italian Journal of Zoology, 64: 91-96.

RotA, E., 1998. Morphology and adaptations of Parergodrilus Reisinger and Hrabeiella Pizl \& Chalupský, two enigmatic soil-dwelling annelids. Italian Journal of Zoology, 65: 75-84.

Rota, E., Martin, P. \& Erséus, C., 2001. Soil-dweling polychaetes: enigmatic as ever? Some hints on their phylogenetic relationships as suggested by maximum parsimony analysis of $18 \mathrm{~S}$ rDNA gene sequences. Contributions to Zoology, 70: 127-138.

Rousset, V., Pleijel, F., Rouse, G. V., Erséus, C. \& SIDALL, M. E., 2007. A molecular phylogeny of annelids. Cladistics, 23: 41-63.

Schmidt, H. \& Westheide, W., 2000. Are the meiofaunal polychaetes Hesioniodes arenaria and Stygocapitella subterranea true cosmopolitan species? - results of RAPD-PCR investigations. Zoologica Scripta, 29: 17-27.

SchwANK, P., 1981. Turbellarien, Oligochaeten und Archianneliden des Breitenbachs und anderer oberheissischer Mittelgebirgsbäche. II. Die Systematik und Autökologie der einzelnen Arten. Archiv für Hydrobiologie Supplement, 62: 86-147.
Schweitzer, A., 1936. Quelques Oligochètes d'Alsace. Bulletin de l'Association Philomathique d'Alsace et de Lorraine, 8: 212-226.

Struck, T., Hessling, R. \& Purschke, G., 2002. The phylogenetic position of the Aelosomatidae and Parergodrilidae, two enigmatic polychaete-like taxa of the 'Polychaeta', based on molecular data from $18 \mathrm{~S}$ rDNA sequences. Journal of Zoological Systematics and Evolutionary Research, 40: 155163.

Westheide, W., 1990. Polychaetes. Interstitial families. Synopses of the British Fauna, New series, 44: 1152.
Recibido, 19-IX-2009

Aceptado, 28-X-2009 Publicado, 31-XII-2009 